\title{
Exploración del riesgo para fluorosis dental en niños de las clínicas odontológicas universidad de Cartagena
}

\section{Exploring the risk of dental fluorosis in children at the Universidad of Cartagena's odontology clinics}

\author{
Katherine M. Arrieta-Vergara ${ }^{1}$, Farith González-Martínez ${ }^{2}$ y \\ Luzmayda Luna-Ricardo ${ }^{1}$
}

1 Departamento de Odontología Preventiva y Social, Facultad de Odontología de la Universidad de Cartagena. katherinearrieta@gmail.com, luzmaydalunar@yahoo.com

2 Departamento de investigación Facultad de Odontología Universidad de Cartagena, Colombia. Correo electrónico: farithgm@hotmail.com

Recibido 30 Septiembre 2010/Enviado para Modificación 22 Mayo 2011/Aceptado 2 Junio 2011

\section{RESUMEN}

Objetivo Describir la prevalencia de fluorosis dental y su relación con factores asociados en niños que acuden a las clínicas de Odontopediatría Universidad de Cartagena.

Métodos Estudio de corte transversal, realizado en la Universidad de Cartagena durante el 2009. Se seleccionó una muestra probabilística aleatoria simple con remplazo. Se examinaron 230 niños utilizando el índice de Thylstrup y Fejerskov y se aplicó una encuesta a las madres de los participantes, que indagaba sobre los factores asociados a la Fluorosis dental. Los datos fueron analizados a partir de proporciones, asumiendo intervalos de confianza del $95 \%$, razones de disparidad (odds ratio) y regresión logística, utilizando el programa STATA $10.0^{\circledR}$. Resultados La prevalencia de la Fluorosis dental fue $66,5 \%$, presentándose un $88,3 \%$ de casos leve y $11,7 \%$ de moderado. No se observaron lesiones severas. Los factores que explican la presencia de Fluorosis dental fueron: cantidad de sal utilizada para preparar los alimentos entre media y una cucharada sopera, primera aplicación de flúor antes de los cuatro años, cantidad de dentífrico usado entre la mitad y la totalidad del cepillo, ingesta de dentífrico durante el cepillado y la ingesta de dentífrico en momentos diferentes al cepillado $\left(p=0.000 ; x^{2}=48,2\right)$.

Conclusiones La prevalencia de Fluorosis en la población de estudio se considera alta, aunque la mayoría de los casos corresponden al estadio leve. Se puede afirmar que esta alteración está influida probablemente por la exposición al mismo tiempo de diferentes fuentes de fluoruros utilizadas para la prevención de la Caries dental.

Palabras Clave: Fluorosis dental, epidemiología, factores de riesgo, fluoruros (fuente: DeCS, BIREME). 


\section{ABSTRACT}

Objective Describing the prevalence of dental fluorosis and its relationship with associated factors in children attending the University of Cartagena's Odontopaediatrics clinics.

Methods A cross-sectional study was carried out at the University of Cartagena during 2009; patients were selected by simple random sampling with replacement (SRSWR). A group of 230 children were examined using the Thylstrup and Ferjerskov index and the participants' mothers were surveyed regarding factors associated with dental fluorosis. STATA 10.0 software was used for analysing the information by percentages, assuming $95 \%$ confidence intervals, disparity ratios and logistic retrogression.

Results There was $66.5 \%$ dental fluorosis prevalence; $88.3 \%$ of cases were mild and $11.7 \%$ moderate. No severe lesions were observed. The factors explaining the presence of dental fluorosis were: the quantity of salt used in preparing food, the first application of fluorine before age 4, the quantity of toothpaste used when brushing, ingestion of toothpaste during brushing and ingestion of toothpaste different to that at brushing time $\left(p=0.000 ; x^{2}=48.2\right)$.

Conclusions The prevalence of fluorosis in the study group was considered to be significantly high, although most cases were very moderate. It can be stated that such alteration was probably influenced by exposure to varied sources of fluoride used in preventing dental caries.

Key Words: Fluorosis, dental, epidemiology, risk factor, fluoride (source: $\mathrm{MeSH}$, $N L M)$.

os suplementos de fluoruros en la dieta y los fluoruros tópicos se han usado desde hace más de 60 años como medida de salud pública para la prevención de la Caries dental. Sin embargo, la ingesta del fluoruros por un periodo de tiempo prolongado en concentraciones más altas que las recomendadas y durante la formación del esmalte dental, puede afectar las funciones de los ameloblastos, alterando los mecanismos de mineralización del diente, llevando a lo que hoy se conoce como Fluorosis dental $(1,2)$. Esta se caracteriza por un incremento en la porosidad de la superficie y en la subsuperficie del esmalte, causando manchas blancas y opacas, estriaciones moteadas, hendiduras transversales y ondulantes de la superficie esmaltada del diente y fosas discontinuas con zonas de sub-desarrollo dental que pueden alterar su morfología (3) y generar extensas fracturas mecánicas de la superficie (4), predisponiendo a la aparición de otras alteraciones como la caries y sensibilidad dental (5-6).

Aunque la incidencia de Caries se ha reducido con los años en edades tempranas, también se ha producido un aumento en la incidencia de fluorosis dental (7). Este aumento se origina probablemente como consecuencia de la 
ingestión excesiva de diferentes fuentes de flúor, lo que ocasiona que las exposiciones totales a este ión puedan ser superiores a las necesarias para prevenir la caries, en especial las formas sistémicas como el flúor en el agua y la sal y las formas tópicas con el uso de dentífricos fluorados $(8,9)$.

En 1998 en el estudio Nacional de Salud Bucal (ENSAB III) (10) se evaluó la presencia de Fluorosis dental en niños y adolescentes, reportándose una prevalencia de $20,3 \%$, posteriormente se han adelantado varios estudios en distintos municipios de Colombia, lo que ha confirmado esta tendencia en todo el territorio nacional (11-14). Estos hallazgos corroboran la necesidad de continuar con la vigilancia epidemiológica de este problema de salud bucal, sobre todo en ciudades del Caribe Colombiano como Cartagena de Indias, donde existe poca explicación sobre la distribución demográfica de este evento y sus factores relacionados. En este sentido, el presente estudio fue realizado con el objetivo de describir la prevalencia de Fluorosis dental y su relación con factores asociados en los niños que acuden a las clínicas de Odontopediatría de la Universidad de Cartagena.

\section{MÉTODOS}

Se realizó un estudio de corte trasversal en 230 niños entre 6 y 11 años de edad que asistieron a las clínicas odontológicas de la Universidad de Cartagena, Colombia, en el segundo periodo de 2009. El tamaño de la muestra fue calculado a partir de una frecuencia esperada del fenómeno en estudio (Fluorosis dental) del $63 \%$ (8), confianza de1 $95 \%$, error tipo I del $5 \%$, error tipo II del $20 \%$ y poder del $80 \%$.

Para la selección de los participantes se utilizó un muestreo probabilístico aleatorio simple con reemplazo, a partir de una población blanco conformada por los niños asistentes a las clínicas Odontológicas de la institución objeto de estudio. La selección fue realizada con ayuda del programa STATA 10.0, generando una lista de números aleatorios. Para poder incluir a los participantes se tuvieron como criterios; residentes en la ciudad de Cartagena durante los cinco primeros años de vida, presentar en completa erupción uno o más pares homólogos de dientes indicados para el examen y que sus padres aceptaran diligenciar un consentimiento informado por escrito. Este aspecto fue soportado por las disposiciones internacionales; declaración de Helsinkimodificación de Edimburgo 2000 y la normatividad para Colombia; resolución 8430 de 1993 del antiguo Ministerio de la Salud. Por otro lado, se excluyeron 
a los niños que tuvieran algún compromiso sistémico o malformación congénita que afecte huesos y dientes, tener en el momento del estudio tratamiento de ortodoncia u ortopedia fija maxilar y aquellos que presentaran caries dental, obturaciones o fracturas dentales en la superficie vestibular examinada. Este proyecto fue avalado por el comité de ética institucional de la universidad de Cartagena, declarándose con riesgo menor del mínimo.

Examen clínico. La presencia y severidad de Fluorosis dental, se evaluó a través del índice Thylstrup y Fejerskov (TFI) $(15,16)$ y un instructivo para identificar los dientes patrones. Se realizó estandarización clínica a partir de un evaluador estándar de oro. Para la selección del mejor examinador se aceptó como criterio un grado de acuerdo igual a 0,80 , a través de la prueba Kappa Cohen, obteniéndose como resultado a dos examinadores. La evaluación de la Fluorosis dental fue realizada en dentición permanente clasificando la apariencia macroscópica de los dientes en relación con la condición histológica del esmalte, a partir de una escala ordinal que va de 0 a 9. El procedimiento se realizó mediante luz artificial, previa remoción de la placa dental mediante profilaxis profesional. Se inspeccionaron las superficies vestibulares, limpias y secas y se determinó la presencia y gravedad de la Fluorosis dental en seis pares de dientes (incisivos centrales y laterales y primeros molares en ambas arcadas).

Cuestionario. Se aplicó una encuesta auto-suministrada con 36 preguntas, 32 fueron estructuradas; 27 politómicas y cinco dicotómicas con única respuesta, que midieron las siguientes variables: características sociodemogràficas de los participantes (edad, género, estrato socioeconómico y procedencia), bajo peso al nacer, consumo de agua en pozo, consumo de agua hervida, preparación de alimentos con cantidad de sal entre media y una cucharada sopera, preparación con sal durante el día entre tres y cinco tipos de alimentos, primera aplicación de flúor tópico antes de los cuatro años, haber recibido entre tres a más topicaciones de flúor al año, aplicación de flúor en cubeta, uso de enjuagatorios bucales fluorados, inicio del cepillado con crema dental fluorada al año o antes, frecuencia del cepillado dental entre tres o más veces al día, uso de dentífrico con alto contenido de flúor, delegación en la supervisión del cepillado dental, no aplicación del dentífrico en el cepillo dental de sus hijos, ingestión accidental de la crema dental durante el cepillado, ingestión de la crema dental en momentos diferentes al cepillado, consumo de leche pulverizada a diario, edad de inicio del consumo de alimentos fluorados a los dos años o menos, frecuencia de consumo de alimentos 
fluorados dos o más veces por semana. Este instrumento fue evaluado por dos jueces para garantizar validez de apariencia. Se tuvo en cuenta la suficiencia de las categorías, la extensión del cuestionario y la pertinencia de cada uno de los tópicos en estudio, lo que permitió hacer las modificaciones necesarias para poder aplicarlo, auditar su buen diligenciamiento y evitar la tasa de no repuesta.

Análisis estadístico. Los datos fueron analizados a partir de estadística descriptiva e inferencial, usando el programa estadístico STATA 10.0® versión para Windows. Se estimó la ocurrencia de la Fluorosis dental y los Factores asociados a través de prevalencias. Para determinar las asociaciones se utilizaron las razones de disparidad (Odds Ratio), con estimadores crudos y ajustados por edad, asumiendo intervalos de confianza del $95 \%$. El análisis multivariable se realizó por regresión logística nominal, evaluándose el efecto de retirar las variables a partir de un límite de significancia de 0,05.

\section{RESULTADOS}

Los 230 sujetos tenían un promedio de edad de 8,2 años $(\mathrm{DE}=1,31)$ y un rango de edades entre 6 y 11 años. En cuanto al sexo, el 48,3\% fueron masculinos y el $51,7 \%$ femeninos, quienes pertenecían a los estratos socioeconómicos bajo (57,0 \%), medio-bajo (34,3\%) y medio $(8,7 \%)$. Con respecto a la procedencia, el $97,8 \%$ de los sujetos tenían residencia actual en la ciudad de Cartagena.

Prevalencia y severidad de fluorosis dental. La prevalencia de la fluorosis dental fue de $66,5 \%$ (IC $95 \%$; 60,4 -72,7), siendo muy similar para la edad, el sexo y estrato social. Para la severidad nivel leve; (TFI 1 y TFI 2) se presentó en el 88,3\%, mientras que el moderado (TFI 3 y TFI 4) en el 11,7 $\%$, no se observaron niveles severos $(\mathrm{TFI}>/=5$ ) (Tabla 1$)$. Los órganos dentarios más afectados fueron los primeros molares con el $60,4 \%$, seguido de los incisivos centrales y los incisivos laterales.

Prevalencia de factores asociados a la fluorosis dental. Los factores asociados de mayor prevalencia fueron la edad de inicio de consumo de alimentos fluorados entre dos años o menos con 88,2 \% (IC $95 \%$; 84,0$92,4)$, frecuencia de consumo de alimentos fluorados dos o más veces por semana con 85,6\% (IC 95\%; 81,1-90,2), ingesta accidental de crema dental durante el cepillado con 76,1 \% (IC 95 \%; 70,5-81,6), preparación con sal 
durante el día entre tres y cinco tipos de alimentos con 76,1 \% (IC 95\%; 70,5-81,6), uso de dentífrico con alto contenido de flúor en ppm (1500 ppm) con 70,4\% (IC $95 \%$; 64,4-76,3) y cantidad de dentífrico utilizada para el cepillado entre la mitad y la totalidad del cepillo con $63,9 \%$ (Tabla 2).

Tabla 1. Prevalencia y severidad de fluorosis de acuerdo a variables socio-demográficas

\begin{tabular}{|c|c|c|c|c|c|c|}
\hline \multirow[b]{2}{*}{ Variables } & \multirow[b]{2}{*}{ número } & \multicolumn{2}{|c|}{ Prevalencia $n=230$} & \multicolumn{3}{|c|}{ Severidad $n=153$} \\
\hline & & $\%^{*}$ & IC 95\% & Leve (\%) & $\begin{array}{c}\text { Moderado } \\
(\%)\end{array}$ & $\begin{array}{c}\text { Severo } \\
(\%)\end{array}$ \\
\hline \multicolumn{7}{|l|}{ Edad } \\
\hline 6 a 7 & 47 & 63,5 & 53,5 & $67(90,5)$ & $7(9,5)$ & $0(0,0)$ \\
\hline 8 a 9 & 81 & 70,4 & 4 & & 16( & 0( \\
\hline 10 a 11 & 25 & 61 & & & 4( & $0(0,0)$ \\
\hline \multicolumn{7}{|l|}{ Sexo } \\
\hline Masculino & 76 & 68,5 & 59 & $0,1)$ & 11( & $0(0,0)$ \\
\hline Femenino & 77 & 64,7 & 3,7 & 103 & $16(13,5)$ & $0(0,0)$ \\
\hline \multicolumn{7}{|l|}{ Estrato social } \\
\hline Bajo & 88 & 67,2 & 59 & $8,6)$ & 15( & $0(0,0)$ \\
\hline Medio- Bajo & 52 & 65,9 & 76,9 & $71(89,9)$ & $8(10,1)$ & $0(0,0)$ \\
\hline Medio & 13 & 65 & $44,0-86,0$ & $16(80,0)$ & $4(20,0)$ & $0(0,0)$ \\
\hline Total & 153 & 66,5 & $60,4-72,7$ & $203(88,3)$ & $27(11,7)$ & $0(0,0)$ \\
\hline
\end{tabular}

Tabla 2. Ocurrencia de factores asociados a Fluorosis en niños atendidos en la clínica de Odontopediatría Universidad de Cartagena 2009

\begin{tabular}{lccc}
\hline \multicolumn{1}{c}{ Factores asociados a fluorosis } & $\begin{array}{c}\text { Numero } \\
\mathrm{n}=230\end{array}$ & $\begin{array}{c}\text { Prevalencia } \\
(\%)\end{array}$ & IC 95\% \\
\hline Bajo peso al nacer & 45 & 19,5 & $14,3-24,7$ \\
Consumo de agua en Pozo & 3 & 1,3 & $0,01-2,7$ \\
Agua hervida para el consumo diario & 122 & 53 & $46,5-59,5$ \\
Sal usada en alimentos (media y/o 1 cucharada) & 107 & 46,5 & $40,0-53,0$ \\
Alimentos preparados con sal (entre tres y cinco) & 175 & 76,1 & $70,5-81,6$ \\
Primera aplicación de flúor antes de los cuatro años & 85 & 36,9 & $30,6-43,2$ \\
Tres o más aplicaciones de flúor en el año & 13 & 5,6 & $2,6-8,6$ \\
Aplicación profesional de flúor en cubeta dental & 87 & 37,8 & $31,5-44,1$ \\
Uso de enjuagatorios fluorados & 40 & 17,4 & $12,4-22,3$ \\
Inicio del cepillado dental del niño al año o antes & 141 & 61,3 & $54,9-67,6$ \\
Frecuencia del cepillado dental tres veces al día & 64 & 30 & $24,0-35,9$ \\
Usa dentífrico con alto contenido de flúor & 162 & 70,4 & $64,4-76,3$ \\
Supervisión del cepillado delegada en otras personas & 22 & 9,5 & $5,7-13,3$ \\
Cepillado dental de su hijo sin dentífrico & 31 & 13,5 & $9,0-17,9$ \\
Cantidad de dentífrico entre la mitad y todo el cepillo & 147 & 63,9 & $57,6-70,1$ \\
\hline Ingesta de crema dental durante el cepillado & 175 & 76,1 & $70,5-81,6$ \\
Ingesta de crema dental sin cepillado & 117 & 50,9 & $44,3-57,3$ \\
Consumo diario de leche pulverizada & 131 & 56,9 & $50,5-63,4$ \\
Consumo alimentos fluorados desde dos años o menos & 203 & 88,2 & $84,0-92,4$ \\
\hline Alimentos fluorados dos o más veces semana & 197 & 85,6 & $81,1-90,2$ \\
\hline
\end{tabular}


Análisis bivariado. Con estimadores crudos, se observaron asociaciones estadísticamente significativas entre la presencia de Fluorosis y la cantidad de sal utilizada para preparar alimentos entre media y una cucharada sopera $(\mathrm{OR}=1,86$; IC $95 \% ; 1,06-3,27)$, primera aplicación de flúor antes de los cuatro años de edad (OR=2,31; IC $95 \%: 1,25-4,25)$, inicio del cepillado dental con crema fluorada al año o antes (OR=1,79; IC 95\%: 1,02-3,14), uso de dentífrico con alto contenido ara adultos durante el cepillado $(\mathrm{OR}=1,93$; IC $95 \%$; 1,07$3,47)$, cantidad de dentífrico utilizado entre la mitad y todo el cepillo $(\mathrm{OR}=1,98$; IC $95 \% ; 1,12-3,48)$, ingesta de dentífrico durante el cepillado $(\mathrm{OR}=4,46$; IC $95 \% ; 2,35-8,45)$, ingesta de dentífrico en momentos diferentes al cepillado $(\mathrm{OR}=3,42$; IC $95 \% ; 1,91-6,12)$ y el consumo de leche pulverizada a diario $(\mathrm{OR}=0,55$; IC $95 \%, 0,31-0,98)$ (Tabla 3$)$.

Al ajustar los estimadores por edad, se presentó significancia para el uso de enjuagatorios fluorados (OR=3,04; IC $95 \% ; 1,58-5,86)$, se aumentó la fuerza estadística para la cantidad de sal utilizada en la preparación de los alimentos, primera aplicación de fluorización antes de los cuatro años de edad y la ingesta de dentífrico en momentos diferentes al cepillado. Además se disminuyó la fuerza estadística para los factores; inicio del cepillado dental al año o antes, uso de dentífrico con alto contenido de flúor en ppm, ingesta de dentífrico durante el cepillado y el consumo de leche pulverizada a diario (Tabla 3).

Análisis multivariable. El modelo que mejor explica la presencia de la Fluorosis en los sujetos de estudio fue el conformado por los factores: cantidad de sal utilizada para preparar los alimentos entre media y una cucharada sopera, primera aplicación de fluorización antes de los cuatro años, cantidad de dentífrico usado entre la mitad y todo el cepillo, ingesta de dentífrico fluorado durante el cepillado y la ingesta de dentífrico en momentos diferentes al cepillado $\left(\mathrm{p}=0.000 ; x^{2}=48,2\right)$ (Tabla 4). A partir de este análisis se realizaron interacciones, observándose aumento de la fuerza estadística al combinar las siguientes variables: ingesta de crema dental durante el cepillado con la frecuencia del cepillado entre tres y más veces al día $(\mathrm{OR}=4,95$; IC $95 \%$; $2,57-9,53)$ y el uso de crema dental con alto contenido de flúor con la frecuencia del cepillado de tres o más veces al día (OR=2,01; IC 95\%; 1,10$3,66)$. 
Tabla 3. Presencia de fluorosis y factores asociados en niños de la clínica de Odontopediatría Universidad de Cartagena 2009

\begin{tabular}{|c|c|c|c|c|c|c|}
\hline \multirow{2}{*}{ Factores asociados a fluorosis } & \multicolumn{2}{|c|}{ Fluorosis } & \multicolumn{2}{|c|}{ Bivariado crudo } & \multicolumn{2}{|c|}{ Bivariado ajustado } \\
\hline & $f$ & $\%$ & OR & IC $95 \%$ & OR a & IC $95 \%$ \\
\hline Bajo peso al nacer & 31 & 68,9 & 1,14 & $0,56-2,3$ & 1,07 & $0,65-1,78$ \\
\hline Consumo de agua en Pozo & 3 & 100 & & & & \\
\hline Agua hervida para el consumo diario & 83 & 68 & $1, \overline{15}$ & $0,66-1,99$ & $0, \overline{9} 7$ & $0,64-1,45$ \\
\hline Sal usada en alimentos (media y/o 1 cucharada) & 78 & 73,8 & $1,86^{*}$ & $1,06-3,27$ & $2,39 \dagger$ & $1,67-3,64$ \\
\hline $\begin{array}{l}\text { Alimentos preparados con sal (entre tres y } \\
\text { cinco) }\end{array}$ & 117 & 66,9 & 1,06 & $0,56-2,01$ & 0,88 & $0,54-1,41$ \\
\hline Primera aplicación flúor antes de los cuatro años & 66 & 77,7 & $2,31 \ddagger$ & $1,25-4,25$ & $2,75 \dagger$ & $1,72-4,39$ \\
\hline Tres o más aplicaciones de flúor al año & 7 & 53,9 & 0,56 & $0,18-1,75$ & 0,68 & $0,29-1,59$ \\
\hline Aplicación profesional de flúor en cubeta dental & 62 & 71,3 & 1,41 & $0,79-2,52$ & 1,41 & $0,92-2,15$ \\
\hline Uso de enjuagatorios fluorados & 31 & 70,5 & 1,91 & $0,86-4,26$ & $3,04 \pi$ & $1,58-5,86$ \\
\hline Inicio del cepillado dental del niño al año o antes & 101 & 71,6 & $1,79^{\star *}$ & $1,02-3,14$ & $1,59^{*}$ & $1,05-2,40$ \\
\hline Frecuencia del cepillado dental tres veces al día & 45 & 65,2 & 0,92 & $0,50-1,66$ & 1,09 & $0,70-1,70$ \\
\hline Usa dentífrico con alto contenido de flúor & 115 & 71 & $1,93+\dagger$ & $1,07-3,47$ & 1,91㧊 & $1,24-2,93$ \\
\hline Supervisión cepillado delegada a otras personas & 14 & 63,6 & 0,86 & $0,34-2,16$ & 0,85 & $0,42-1,69$ \\
\hline Cepillado dental de su hijo sin dentífrico & 24 & 77,4 & 1,86 & $0,76-4,53$ & 1,78 & $0,92-3,43$ \\
\hline $\begin{array}{l}\text { Cantidad dentífrico entre la mitad y todo el } \\
\text { cepillo }\end{array}$ & 106 & 72,1 & 1,98शा & $1,12-3,48$ & $1,97 \rrbracket$ & $1,30-2,98$ \\
\hline Ingesta de crema dental durante el cepillado & 131 & 74,9 & $4,46 \dagger$ & $2,35-8,45$ & $4,16 \dagger$ & 2,61-6,64 \\
\hline Ingesta de crema dental sin cepillado & 93 & 79,5 & $3,42 \dagger$ & $1,91-6,12$ & $3,63 \dagger$ & $2,36-5,60$ \\
\hline Consumo diario de leche pulverizada & 80 & 61,1 & $0,55^{\star *}$ & $0,31-0,98$ & $0,50 \pi$ & $0,32-0,76$ \\
\hline Alimentos fluorados desde dos años o menos & 136 & 67 & 1,19 & $0,51-2,74$ & 0,99 & $0,52-1,87$ \\
\hline $\begin{array}{l}\text { Alimentos fluorados dos o más veces por } \\
\text { semana }\end{array}$ & 131 & 66,5 & 0,99 & $0,45-2,16$ & 0,9 & $0,48-1,66$ \\
\hline
\end{tabular}

Tabla 4. Modelo de regresión logística para la presencia de Fluorosis y Factores asociados en niños de la clínica de Odontopediatría Universidad de Cartagena

\begin{tabular}{lccc}
\hline \multirow{2}{*}{ Factores Asociados a Fluorosis } & \multicolumn{2}{c}{ Multivariado a } & \\
\cline { 2 - 3 } & ORT & IC 95\% & Significancia \\
\hline Sal usada en alimentos (media y/o 1 cucharada) & 2,34 & $1,23-4,45$ & 0.009 \\
Primera aplicación de flúor antes de los cuatro años & 2,16 & $1,11-4,19$ & 0.023 \\
Cantidad de dentífrico entre la mitad y todo el cepillo & 1,91 & $1,02-3,60$ & 0.044 \\
Ingesta de crema dental durante el cepillado & 3,42 & $1,68-6,97$ & 0.001 \\
Ingesta de crema dental sin cepillado & 2,77 & $1,43-5,35$ & 0.002 \\
\hline
\end{tabular}

a Significancia del modelo $\left(p=0.000 ; x^{2}=48,2\right)$; TOR Ajustados por regresión

\section{DISCUSIÓN}

Los resultados del presente estudio provienen de procedimientos, métodos e instrumentos clínicos validos y confiables que garantizan la utilización de esta información en el contexto en el que fueron aplicados, siendo utilizado el índice TFI (17-19) para el examen de fluorosis, por su confiabilidad, sensibilidad y precisión, (20) y especialmente por la capacidad clínica que tienen los examinadores al diferenciar entre la variedad de cambios asociados con los signos tempranos y las formas más severas de la Fluorosis dental (21). 
La prevalencia de la fluorosis dental observada en el presente estudio es considerada alta, producto probablemente del exceso de exposición a diferentes fuentes de fluoruros como medida preventiva para la caries dental, estos hallazgos son similares a los reportados en el 2003 por Ramírez (11) en niños del municipio de Frontino (Antioquia) (67\%), a los encontrados en 2007 por el mismo autor en instituciones educativas privadas de Medellín $(79,1 \%),(12)$ y en instituciones educativas públicas de Medellín (81\%) (13). Además, en escolares de instituciones educativas privadas de la ciudad de Bucaramanga en 2003, Concha (14) encontró presencia de Fluorosis en un $71,4 \%$. Estos hallazgos ponen en evidencia el aumento de la ocurrencia a nivel nacional $(17,18)$, hallazgo que también ha sido reportado en numerosos estudios internacionales $(22,23)$.

De acuerdo a los factores asociados a Fluorosis, los de mayor prevalencia fueron el inicio y la frecuencia del consumo de alimentos fluorados, lo que puede explicarse por ser la ciudad de Cartagena un puerto marítimo, en donde la preferencia en la dieta culturalmente ha incluido desde tempranas edades el consumo de pescado en diferentes especies y presentaciones. Por otro lado, también tuvo un peso importante todos los factores relacionados con el uso de cremas dentales (ingesta accidental de crema dental durante el cepillado, uso de dentífrico con alto contenido de flúor en ppm, cantidad de dentífrico utilizada para el cepillado entre la mitad y la totalidad del cepillo y frecuencia del cepillado dental entre tres o más veces al día). Estos han sido reportados como exposiciones de importante peso específico, sobre todo por el poco control que los padres tienen sobre el cepillado dental de sus hijos en estas edades (24).

Entre los factores que presentaron mayor fuerza de asociación con la Fluorosis dental, se destaca el consumo de sal en la preparación de alimentos, el cual se considera esencial dentro de la dieta de la costa atlántica colombiana, este aumento en la cantidad de consumo diario puede acentuar la absorción del flúor en el organismo, causando efectos adversos en la etapa de formación de los gérmenes dentarios (25). En este mismo sentido, Martignon (21) en niños de planteles educativos privados y públicos de Bogotá encontró mayor fuerza de asociación para la primera aplicación de flúor antes de los cuatro años de edad, esta práctica es muy utilizada por algunos Odontólogos en el consultorio dental para la prevención de la caries a temprana edad, no obstante la dificultad que existe para el control de la ingesta accidental de este producto a tempranas edades, esta se considera una fuente adicional de absorción de flúor en nuestro medio (26). 
Por otra parte, la exposición al flúor a través de las cremas dentales, ha sido reportada en numerosos estudios a nivel internacional como riesgo para fluorosis $(16-19,25,26)$. Tabari (27) y Mascarenhas (28), reportaron que los niños que usaban cremas dentales fluoradas ( $>1,200$ ppm de flúor) tienen entre 1,6 y 1,83 veces mayor riesgo en comparación con niños que usan cremas dentales con baja concentración de flúor. Por otro parte, Pendrys y Katz (19) y Osuji (26) coinciden con los resultados del presente estudio en referencia a que los niños que inician su cepillado antes de los dos años tienen 6,3 y 11 veces respectivamente, mayor probabilidad de presentar fluorosis.

Con la evidencia sustentada a partir de los resultados del presente estudio, se confirma que los niños en edades tempranas se exponen a diferentes fuentes de fluoruros, las cuales son recomendadas y utilizadas indiscriminadamente por Odontólogos y adultos para evitar la presencia de la Caries dental. Es claro que en estas edades existe poco control en la expectoración de los residuos de la crema dental durante el cepillado, así mismo se ha demostrado que los niños entre 1,5 y 2,5 años de edad ingieren entre el 64,3\% y el 83, $9 \%$ de la crema dental que utilizan (29), e incluso aún a los cinco años de edad se ingiere alrededor del $30 \%$ durante esta actividad (30). Por otro lado, el riesgo se incrementa si además de la ingesta del dentífrico, la frecuencia del cepillado es mayor a dos veces al día (31), si realizan el cepillado con crema dental con alto contenido de flúor $(<1,200 \mathrm{ppm})$ (31) o si se coloca gran cantidad de dentífrico en el cepillo (24).

El modelo encontrado a partir del análisis multivariable en el presente estudio puede ser importante para utilizar el peso específico de cada uno de los cinco factores involucrados y lograr que esto influya en las decisiones de Odontólogos y padres de familia, para evitar exposiciones innecesarias a las fuentes de flúor. En este sentido, se concluye que la alta prevalencia de Fluorosis dental en esta población está influida por exposiciones a los fluoruros originadas desde la dieta y la ingestión de las cremas dentales durante el cepillado, lo que puede considerarse como un importante conocimiento para evitar que en niños con estas características se siga aumentando la presencia de esta alteración, el cual puede ser reconocido como un problema de salud pública *

Agradecimientos: A todo el personal académico y administrativo de la Facultad de Odontología, Universidad de Cartagena, Colombia.

Conflictos de Interés: Ninguno 


\section{REFERENCIAS}

1. Petersen PE. The world oral health report 2003: continuous improving of oral health in the 21st century; the approach of the WHO Global Oral Health Programme. Community Dent Oral Epidemiol. 2003; 31(Supl 1):3-23.

2. Fejerskov O, Baelum V, Richards A. Dose-response and dental fluorosis. In: Fejerskov O, Ekstrand J, Burt BA (Ed.). Fluoride in dentristry. 2nd Ed. Copenhagen: Musksgaard. 1996; p. 153-66.

3. DesBesten PK, Keels MA, Heller KE. The effect of dental fluorosis to the use of fluoride supplements. Community Dent Oral Epidemiol. 1999; 27:41-7.

4. Fejerskov MJ, Richards A, Baelum V. Dental tissue effects of fluoride. Adv Dent res. 1994; 8(1): $15-31$.

5. Cunha-Cruz J, Nadanovsky P. Dental Fluorosis. Increases caries risk. Journal of evidence based dental practice. 2005; 5: 170-171.

6. Thylstrup A, Fejerskov O. Clinical appearance of dental fluorosis in permanent teeth in relation to histologic changes. Community Dent Oral Epidemiol. 1978; 6:315-328.

7. Cochran JA, Ketley CE, Árnadóttir IB, Fernandes B, Koletsi-Kounari H, Oila A-M, et al. A conparison of the prevalence of flourosis in 8-year-old children of seven european study sites a standardized methodology. Community Dent Oral Epidemiol. 2004; 32 (suppl 1):28-33.

8. Sánchez H, Parra JH, Cardona D. Fluorosis dental en escolares del Departamento de Caldas, Colombia. Biomédica. 2005; 25:46-54.

9. Quanyong X, Minghao Z, Ming W, Xinya Z, Li L, Jiuning H. Relationships between daily total fluoride intake and dental fluorosis and dental caries. Journal of Nanjing Medical University. 2009; 23: 33-39.

10. Ministerio de Salud, República de Colombia. Centro Nacional de Consultoría CNS: IIIENSAB III; Tomo VII. Bogotá: Lito Servicios ALER; 1999.

11. Ramírez BS, Franco AM, Sierra JL, López RV, Alzate T, Sarrazola AM, Pimienta C, Morales C. Fluorosis dental en escolares y exploración de factores de riesgo. Municipio de Frontino. Rev Fac Odontol Univ Antioq. 2006; 17(2):26-33.

12. Ramírez B, Franco A, Gómez A, Corrales D. Fluorosis dental en Escolares de Instituciones Educativas Privadas. Medellin, Colombia: 2007. Rev. Facultad de Odontología de Universidad de Antioquia. 2010; 21(2):170-176.

13. Ramírez B, Franco A, Ochoa E. Fluorosis Dental en Escolares de 6 a 13 Años de Instituciones educativas Públicas de Medellín, Colombia. 2006. Rev salud publica (Bogotá). 2009; 11(4):631-640.

14. Concha S, Celedón Y, Vera W, Poveda E, Muñoz C, Vergel T, et al. Prevalencia de fluorosis dental en escolares de 6 a 15 años de edad de la zona urbana de Bucaramanga. Rev Ustasalud. 2003; 2:73-82.

15. Thylstrup A, Fejerskov O. Textbook of Cariology. Munksgaard International Publishers Lt.; 1986.

16. Fejerskov O, Baelum V, Manji F, Moller IJ. Deltal fluorosis. A Handbook for Health Workers. Copenhagen: Munksgaard; 1988. pp. 44-50.

17. Villena RS. An investigation of the transverse technique of dentifrice application to reduce the amount of fluoride dentifrice for young children. Pediatr Dent. 2000; 22:312-7.

18. Puppin-Rontani RM, Kassawara AB, Rodriguez CR. Influence of socioeconimic level and dentrifice brand on the oral higiene habits and fluoride dentifrice ingestion. JClin Pediar Dent. 2002; 26:319-325.

19. Pendry DG, Katz RV. Risk factor enamel fluorosis in optimally fluoridated children born after the US manufactures decition to reduce the fluoride concentration of infant formula. Am J Epidemiol. 1988; 148(10):967-74. 
20. Mabelya L, Van't Hot MA, Köning KG, Van Palenstein WH. Comparison of two índices of dental fluorosis in low, moderate and high fluorosis Tanzania populations. Community Dent Oral Epidemiol. 1994; 22:415-20.

21. Martignon S, Granados O. Prevalencia de fluorosis dental y análisis de asociación a factores de riesgo en escolares de Bogotá. Rev Cient U. El Bosque. 2002; 8(1):19-27.

22. Molina N, Castañeda R, Hernández JC, Robles G. Prevalencia de fluorosis dental en escolares de una delegación política de la Ciudad de México. Rev Mex Pediatr. 2005; 72(1): 13-16.

23. Vallejos-SánchezA, Medina-Solís CE, Casanova-Rosado JF, Maupomé G, Minaya-Sánchez M, Pérez Olivares S. Dental fluorosis in cohorts born before, during and after the national salt fluoridation program in a community in Mexico. Acta Odontol Scand. 2006; 64: 209-213.

24. Franco AM, Martignon S, Saldarriaga A, González MC, Arbeláez MI, Ocampo A, Luna LM, Martínez- Mier EA, Villa AE. Total fluoride intake in children aged 22-35 months in four Colombian cities. Community Dent Oral Epidemiol. 2005; 33:1-8.

25. Hodge $\mathrm{HC}$. The concentration of fluorides in drinking wáter to give the point of minimun caries whith máximum safety. JADA 1950; 40: 436-439, 1950. In: Morgan L, Allfred E, Tavares M, Bellinger, Needieman. Investigation of the possible associations between fluorosis, fluoride exposure and childhood behavior problems. Ped Dent. 1988; 20: 244-251.

26. Osuji OO, Leake JL, Chipman ML, Nikiforuk G, Locker D, Levine N. Risk factors for dental fluorosis in a fluoridated community. J Dent Res. 1988; 67 (12): 1488-92.

27. Tabari ED, Ellwood R, Rugg-Gunn AJ, Evans DJ, Davies RM. Dental fluorosis in permanentincisor teeth in relation to water fluoridation, social deprivation and toothpaste use in infancy. Br Dent J. 2000; 189:216-20.

28. Mascarenhas AK. Risk factor for dental fluorosis: a review of the recent literature. Pediatr Dent. 2000; 22:269-77.

29. Cochran JA, Ketly CE, Duckworth RM, Van Loveren C, Holbrook WP, Seppä L, et al.Development of a standardize method for comparing fluoride ingested from toothpaste by $1,5-3,5$ years-old children in seven European countries. Part 2: Ingestion result. Community Dent Oral Epidemiol. 2004; 32 (supl 1):47-53.

30. Abanto J, Rezende K, Salazar S, Alves F, Celiberti P, Ciamponi A. Dental fluorosis: Exposure, prevention and managment. Med oral Patol Oral Cir Bucal. 2009; 14(2): E103-7.

31. Bottenberg P, Deeclerck D, Ghidey W, Bogaerts K, Vanobbergen J, Martens L. Prevalence and determinants of enamel fluorosis in Flemish schoolchildren. Caries Res. 2004; 38:20-28. 